\title{
Temas emergentes y conclusiones en la investigación acerca de los equipos y el trabajo de equipo*
}

Deborah DiazGranados \& Eduardo Salas

Los equipos constituyen una parte crítica de las organizaciones actuales. En este artículo proporcionamos una revisión de los temas importantes que se encuentran en la literatura acerca de los equipos. Se examinan los componentes necesarios del trabajo de equipo y los mecanismos de coordinación que promueve la efectividad. Se incluye una discusión de las estrategias de entrenamiento de equipo que se han desarrollado para apuntar hacia el éxito en los equipos. Concluye con una propuesta de diversas rutas que pueden ser consideradas por los investigadores de equipos para contestar aquellas preguntas que permanecen sin respuesta.

Equipos / Trabajo en Equipo / Entrenamiento e investigación de Equipos.

\section{Emerging topics and findings the science on teams and teamwork}

Teams are a critical part of organizations today. In this paper we provide a review of the critical topics found in the team literature. This paper reviews the necessary teamwork components and coordinating mechanisms that promote effectiveness. Included is a discussion of the team training strategies that have been developed in order to target success in teams. The paper concludes with several avenues that can be taken by team researchers to address the questions that remain unanswered.

Teams / Teamwork / Training and investigation of Teams.

* Traducción del inglés por Ricardo Braun, revisado por Arturo Solf. 


\section{INTRODUCCCIÓN}

El estudio de pequeños grupos ha tenido lugar desde el inicio del siglo XX (Durkheim, 1946; Triplett, 1898). Sin embargo, las investigaciones en el pasado frecuentemente se centraban en los grupos que surgían naturalmente (por ejemplo: amigos, familia) y en el estudio de las dinámicas entre los individuos. La psicología industrial/organizacional y la literatura sobre el comportamiento organizacional distinguen la investigación sobre los equipos definiendo al equipo como un tipo especial de grupo. Más específicamente, los equipos se definen como un conjunto distinguible de dos o más individuos que interactúan en forma dinámica, adaptativa e interdependiente, comparten metas o propósitos comunes y tienen roles específicos o funciones para realizar (Salas, Dickinson, Converse \& Tannebaum, 1992).

El estudio de los equipos es un tema bastante investigado en la psicología y en los negocios, si tomamos en cuenta la cantidad de publicaciones sobre el particular. Sin embargo, esta aseveración no tiene por finalidad paralizar la investigación sobre equipos; al contrario, creemos que hay nuevos constructos para investigar, y los conocidos necesitan ser refinados sobre la base de la investigación.

En este trabajo ofrecemos nuestras impresiones y revisamos la investigación sobre equipos de trabajo y trabajo en equipo. El propósito del artículo es doble. En primer lugar, queremos proporcionar al lector una revisión de los temas más importantes sobre equipos y trabajo de equipo, a través del análisis de los artículos más destacados y citados de esta temática. En segundo lugar, ofrecemos a estudiosos y personas interesadas una orientación hacia el rumbo que pueden tomar sus investigaciones en el futuro.

Sin embargo, antes de saber hacia dónde dirigirnos en la investigación, necesitamos conocer dónde nos encontramos y cómo llegamos aquí. Con esto en mente, este artículo ha sido organizado de tal modo que podremos: 1) examinar varias taxonomías de equipos, que han sido presentadas y utilizadas en la investigación de estos, 2) discutir las competencias críticas del trabajo en equipo, 3) examinar algunas intervenciones que apuntan hacia los procesos críticos de trabajo en equipo, y 4) proporcionar una breve revisión de las preguntas aún sin responder en la investigación de equipos. Antes de empezar, presentaremos al lector una breve reseña histórica de los equipos y de la dinámica de grupo, así como una definición de equipos y de trabajo de equipo.

\section{EQUIPOS Y TRABAJO DE EQUIPO}

\section{Historia}

Hay dos formas de organizar a las personas para el trabajo. Uno es el método 
basado en el modelo establecido por los militares, de jerarquía estructurada. Más específicamente, hay un individuo que es el superior y otro el subordinado. El superior es el que da las órdenes y el subordinado el que las acata. El segundo método tiene su base en el modelo tradicional del agro y en el sistema del gremio manufacturero. Es el que se trabaja en pequeños grupos en donde el trabajo es una actividad colectiva (Levi, 2001).

Con el nacimiento de la administración científica durante la Revolución Industrial, la teoría de la administración que analizaba e integraba los procesos del flujo laboral para mejorar la productividad produjo trabajos más simplificados. La jerarquía estructurada fue utilizada para que los sistemas pudieran operar en forma más eficiente (Taylor, 1911). Era un sistema que funcionaba bien pero creó problemas, como la desmotivación de los empleados; los sistemas eran más complejos e inflexibles al cambio y era difícil incorporar nuevas metas, como la calidad, más allá de la sola eficiencia.

Se empezó a cuestionar el método de la administración científica en las décadas de 1920 y 1930, y los trabajadores empezaron a organizarse por sí mismos. El incremento de la membresía en los sindicatos y el desarrollo de otras organizaciones laborales demostraban que el compromiso de por vida con el trabajo, lo cual era muy usual en aquella época, se empezaba a deteriorar. Hubo problemas en las relaciones humanas en los centros laborales y esto condujo al interés por los aspectos sociales del trabajo (Levi, 2001). Más aún, los estudios Hawthorne (Mayo, 1933) condujeron a lo que se ha venido a llamar movimiento de las relaciones humanas, que se enfocaba en los aspectos sociales del trabajo, tal como las normas grupales.

Los investigadores (psicólogos organizacionales e ingenieros industriales) en las décadas de 1960 y 1970 refinaron el uso de los equipos en el trabajo con el desarrollo de la Teoría de los Sistemas Sociotécnicos (TSS) (Appelbaum \& Batt, 1994). La TSS proporciona un método para determinar la mejor forma de organizar a los individuos en el trabajo basándose en sus tareas. Esta teoría sostiene que los equipos son más eficaces si sus tareas son interdependientes y requieren coordinación. Por lo tanto, aquellos individuos que necesitaban coordinar entre sí para completar su tarea estaban organizados de tal forma que trabajaban cerca el uno del otro.

En la década de 1970 también ocurrió la introducción de los círculos de calidad, lo cual condujo al desarrollo de la Administración de la Calidad Total en la década de 1980. La industria japonesa se encontraba en crecimiento; sus productos, de gran calidad y precios bajos, estaban distribuidos alrededor del mundo. Los expertos en negocios de diversos países se encon- 
traban impresionados con el método japonés, su producción de gran calidad, que al mismo tiempo mantenía bajos costos, y viajaron al Japón para ver cómo se lograban estas metas de producción intensa. En sus visitas conocieron los círculos de calidad y los grupos paralelos de producción, donde los trabajadores y los supervisores laboraban juntos para analizar los problemas y desarrollar soluciones a las dificultades de la calidad en el proceso manufacturero.

Con esta información empezó un movimiento hacia los círculos de calidad y la Administración de la Calidad Total, cuyas prácticas impulsaron el énfasis en los equipos (Levi, 2001). Además, hay otras características organizacionales que han mantenido $\mathrm{y}$ desarrollado dicho interés. Por ejemplo, el avance de la tecnología, que permitió un mayor trabajo de equipo, la tendencia a las organizaciones planas, los procesos de reingeniería de los negocios y la globalización de los mercados (Mohrman, Cohen \& Morhman, 1995). Aun cuando el énfasis en los equipos para mejorar la eficacia laboral comenzó como resultado de los círculos de la calidad y la Administración de la Calidad Total, las tendencias mencionadas — que cambiaron el formato del trabajo y de las organizaciones - han promovido el uso continuado de los equipos. Poniendo fin a los aspectos históricos sobre el tema, a continuación se proporciona una definición de los equipos y los trabajos de equipo.

\section{Definición de equipos y de trabajo de equipo}

De acuerdo con Salas y CannonBowers (2000), equipo es "el conjunto de dos o más individuos que deben interactuar y adaptarse a objetivos específicos, compartidos y valorados" (p. 313). Las tareas que el equipo debe realizar deben contener interdependencia, de tal modo que ningún individuo pueda completar la tarea por sí solo, y el conocimiento necesario para realizar la tarea está disperso entre los miembros. Otras características que Salas y Cannon-Bowers (2009) utilizan para delinear los equipos son los roles que están definidos tanto en el nivel funcional como en el jerárquico, comparten dos o más fuentes de información y tienen un tiempo limitado.

Antes de continuar, se debe hacer una importante distinción entre equipos y grupos. Los equipos difieren de los grupos en razón de su grado de interdependencia, su estructura y sus requisitos (Goodman, Ravlin \& Schminke, 1987; Salas \& Cannon-Bowers, 2000). Adicionalmente, Salas y Cannon-Bowers (2001) señalan que los equipos y grupos también difieren con respecto a su conocimiento y habilidades, necesidad de retroalimentación crítica del equipo para el éxito y el de- 
sarrollo de objetivos compartidos, expectativas y visión. Aun cuando es común ver que los términos equipo y grupo son usados en forma intercambiable, creemos que los equipos tienen características únicas que la mayoría de grupos pequeños no tienen.

Lo que ocurre entre los miembros de un equip es lo que se denomina "trabajo de equipo"; es decir, la habilidad de las personas que integran un equipo para trabajar juntos, comunicarse con efectividad, anticipar y satisfacer las necesidades de los demás e inspirar confianza, resultando todo esto en una acción colectiva coordinada (Salas \& CannonBowers, 2001). McIntyre y Salas (1995) sostienen que el trabajo en equipo es un componente esencial y requiere una explicación de cómo procede. Diversas características conductuales del trabajo en equipo han sido objeto de investigación y discusión en la literatura. Más adelante una revisión de estas características será discutida. Ahora que hemos definido los dos conceptos básicos, pasaremos a una breve discusión de los diversos tipos de equipo que existen, $\mathrm{y}$, posteriormente, se analizarán las competencias de equipo y los componentes del trabajo de equipo.

\section{TIPOS DE EQUIPO}

Se pueden encontrar equipos en el cuidado de la salud, en la aviación, en la investigación y desarrollo, en el márketing, en la política, en la actividad mili- tar y en otros contextos. Está claro que no todos los equipos realizan el mismo tipo de trabajo, y es por ello que en la literatura sobre el tema se observan varias taxonomías de equipos (por ejemplo, Cohen \& Bailey, 1997; Sundstrom, De Meuse \& Futrell, 1990). En un impactante artículo Sundstrom y colegas usaron un enfoque ecológico para analizar los factores en la efectividad de los equipos de trabajo. A través de su enfoque identificaron aplicaciones de los equipos de trabajo, creando así una taxonomía de tipos de equipos. Cohen y Bailey identificaron tendencias en los equipos para lograr ser eficaces en función de la tarea, factores de diseño, factores ambientales, procesos internos/ externos, y de los rasgos del grupo. Presentaron su discusión en el contexto de cuatro tipos de equipos. En los siguientes párrafos se presenta una breve reseña de los trabajos de Sundstrom et al, así como de Cohen y Bailey.

Sundstrom et al. (1990) veían a los equipos como pertenecientes a una sola clase. Sin embargo, determinaron que existen cuatro categorías generales de equipos de producción: a) Consejo y Participación; b) Producción y Servicio; c) Proyectos y Desarrollo, y d) Acción y Negociación. Bajo cada tipo de equipo existen individuos interdependientes que comparten responsabilidad, pero que difieren en los tipos de tareas que realizan. Por ejemplo, los equipos de Consejo y Participación se encuentran con mayor frecuencia en las 
organizaciones bajo el nombre de círculos de control de calidad (Cole, 1982). El propósito de estos equipos es reunirse para identificar las áreas de mejora. Sin embargo, el alcance de estos tipos de equipos es usualmente limitado.

Aquellos equipos que se encuentran bajo el nombre de Producción y Servicio utilizan la tecnología para generar productos; por ejemplo, los equipos que laboran en una cadena de montaje para ensamblar o instalar los componentes de una computadora. Estas clases de equipos eligen a su propio líder y se dividen las tareas, y sus miembros son empleados de primera línea. También se les conoce con otros nombres, como equipos autoadministrados o equipos autorregulados (Hackman, 1986; Pearce \& Ravlin, 1987). Más allá del nombre, sus características son similares.

Los grupos de ingenieros o diseñadores que forman equipos estarían bajo la categoría de equipos de Proyectos y Desarrollo. Sundstrom et al. (1990) consideraron que los resultados de estos equipos son complejos y únicos, lo cual origina que para el logro de sus objetivos requieran un periodo de vida más largo que los equipos de diferente tipo.

El último tipo de equipo analizado por Sundstrom et al. (1990) fue el de Acción y Negociación. Constituyen ejemplos de este los equipos deportivos, las unidades militares de comba- te y las tripulaciones de vuelo. Lo que más los diferencia es el tener que actuar generalmente bajo circunstancias impredecibles. Además, los individuos a menudo tienen roles muy especializados; por ejemplo, en una unidad militar de combate que efectúa una misión de reconocimiento los individuos tienen tareas especializadas basadas en su rango y posición.

Cohen y Bailey (1997) presentaron su revisión en el contexto de cuatro tipos de equipos que se pueden encontrar en las organizaciones. Estos son: 1) Equipos de Trabajo; 2) Equipos Paralelos; 3) Equipos de Proyectos; y 4) Equipos de Administración. Es importante no perder de vista que muchos investigadores (Katzenbach \& Smith, 1993; Mohrman et al., 1995; Sundstrom et al., 1990, entre otros) han proporcionado diversas taxonomías de equipos para ilustrar que no todos son iguales. Cohen y Bailey proporcionaron una taxonomía general de los tipos de equipos que coinciden con otras taxonomías.

Los Equipos de Trabajo, tal como los definen Cohen y Bailey (1997), son los más comunes, y generalmente responsables de la fabricación de productos tangibles o de la entrega de un servicio, similar a los equipos de Producción y Servicio de Sundstrom et al. Cohen (1993) describió la pertenencia de sus miembros como estable y bien definida. Algunos ejemplos de estos tipos son los equipos de mineros y los 
de auditoría.

Se denomina Equipo Paralelo (similar a los equipos de Consejo y Participación de Sundstrom et al.) aquel que reúne a individuos de diferentes unidades de trabajo para realizar una función especial, para la cual la organización no estaba preparada. Debido a lo particular de su función, generalmente estos equipos tienen una limitada autoridad y usualmente trabajan estrechamente con la alta gerencia para realizar los trabajos asignados, tales como la solución a un problema o actividades orientadas a la mejora.

El tercer tipo de equipo presentado por Cohen y Bailey (1997) son los Equipos de Proyecto, que se parece a los equipos de Proyecto y Desarrollo de Sundstrom et al. (1990). Estos equipos son limitados en el tiempo, puesto que producen resultados una sola vez, como el lanzamiento de un nuevo producto para su comercialización, o un nuevo sistema de información. Sus tareas no son repetitivas y requieren la aplicación del juicio y pericia de los miembros. A fin de poseer la pericia necesaria es a menudo interfuncional; al convocar a miembros de diferentes unidades funcionales, el conocimiento y la pericia es lo suficientemente diversa como para lograr la tarea encomendada.

El cuarto tipo de equipo discutido por Cohen y Bailey (1997) son los Equipos de Administración, que están formados por individuos con roles de liderazgo en una organización. Este tipo de equipos tiene como responsabilidad proporcionar la dirección a sus subunidades, o trabajan en forma conjunta con el fin de mantener o crear una estrategia para hacer funcionar la organización. Por ejemplo, es común encontrar un equipo ejecutivo de administración en la cabeza de la organización, que es responsable de la elaboración de la dirección de la organización a través de la identificación de las oportunidades y dificultades externas. Otra de sus funciones es la determinación de la dirección estratégica de la empresa (Wiersema \& Bantel, 1992). Estos tipos de equipos usualmente responden ante los accionistas de una compañía o al directorio de esta.

\section{COMPETENCIAS DE EQUIPO $Y$ COMPONENTES DEL TRABAJO DE EQUIPO}

\section{Competencias de equipo}

Cannon-Bowers, Tannenbaum, Salas y Volpe (1995) definieron las competencias del equipo como el Conocimiento, Habilidades y Actitudes (CHA) que se requieren para un trabajo efectivo en equipo. El conocimiento se refiere a la comprensión necesaria de hechos, información y conceptos relacionados con el cumplimiento de la tarea. Las habilidades son procedimientos conductuales y cognitivos necesarios para el desempeño de la tarea. Las actitudes son los componentes afectivos de una tarea. Estas competencias son los cimientos 
del trabajo efectivo en equipo.

Cannon-Bowers et al. (1995) explicaron, además, cómo las competencias de equipo pueden ser identificadas en dos categorías, una referida a la tarea y la otra al equipo. De este modo, una competencia puede ser específica o genérica a la tarea, o bien al equipo. Por ello, Cannon-Bowers et al. sostienen que la variación de la efectividad de un miembro del equipo dependerá de si los CHA poseídos son específicos o genéricos en relación con la tarea o el equipo. Las competencias específicas de la tarea son particulares a esta. Las competencias genéricas de la tarea son generalizables. Por ejemplo, las habilidades interpersonales y de comunicación son aplicables en muchas tareas. Las competencias específicas de equipo están vinculadas a un conjunto de miembros del equipo. Por ejemplo, el conocimiento compartido de un equipo puede cambiar si algunos están ausentes o son reemplazados. Las competencias genéricas de equipo son aquellas que se pueden generalizar a entornos con diferentes miembros de equipo. Lo que Cannon-Bowers et al. presentaron fue una definición de las competencias distintas de las competencias individuales.

\section{Componentes del trabajo de equipo}

Salas, Sims y Burke (2005) proporcionaron un marco teórico de lo que denominaron los Cinco Grandes (Big Five) del trabajo en equipo. Sostienen que el trabajo en equipo podía ser dividido en cinco componentes centrales: Liderazgo de Equipo, Monitoreo Mutuo del Desempeño, Comportamiento de Respaldo, Adaptabilidad y Orientación de Equipo. Salas et al. ofrecen este marco teórico como una taxonomía práctica que incluye no solo los componentes centrales que afectan el rendimiento de equipo, sino también los mecanismos de coordinación necesarios para una interacción útil de los cinco factores en mención.

Los mecanismos de coordinación consisten en el desarrollo de modelos mentales compartidos (Stout, CannonBowers, Salas \& Milanovich, 1999), logro de confianza mutua (Webber, 2002), y compromiso en una comunicación interconectada (closed-loop communication) (McIntyre \& Salas, 1995). Adicionalmente, Salas et al. explicaron que la habilidad de un equipo para utilizar los Cinco Grandes y los mecanismos de coordinación fluctuará conforme avance hacia la tarea y con la experiencia de trabajar como equipo a lo largo del tiempo. Pasaremos ahora a la discusión de los componentes específicos del trabajo en equipo (los Cinco Grandes) y los mecanismos de coordinación.

- Liderazgo. Salas et al. (2005) consideran que el liderazgo de equipo es uno de los Cinco Grandes porque el fracaso del líder de equipo para guiarlo apropiadamente puede ser la 
razón primordial de la ineficacia del equipo (Stewart \& Manz, 1995). Son tres las principales funciones que tiene el líder:

Guía el Modelo Mental Compartido, es decir, tiene un rol en su creación y mantenimiento. Desde la etapa de formación del equipo el líder fomenta una exacta comprensión compartida de los objetivos, las limitaciones potenciales, los roles de cada miembro, y los recursos disponibles para el equipo (Salas et al., 2005).

Facilita la adaptabilidad del equipo, asegurándose de que no sean sorprendidos por los cambios en su entorno. Adicionalmente, el líder usa el ambiente externo para ayudar en la coordinación e interacciones de los comportamientos del equipo (Roby, 1968; Zaccaro, Marks, O'ConnorBoes \& Costanza, 1995).

Establece las expectativas conductuales y de rendimiento (Salas, Burke \& Stagl, 2004). El líder es el que crea el clima de equipo y alienta conductas, tales como el monitorio mutuo del rendimiento, comportamiento de respaldo y adaptabilidad.

- Monitoreo mutuo del rendimiento. Es la habilidad de los miembros de equipo para observar el comportamiento, uno del otro, mientras se realiza la tarea, para asegurarse de que todo funciona adecuadamente (McIntyre \& Salas, 1995). La inves- tigación demuestra que los individuos que experimentan una sobrecarga laboral incurren en más errores (Roby \& Lanzetta, 1957), y el monitoreo mutuo del rendimiento puede ser una solución para prevenir la ejecución de errores. Existen dos prerrequisitos para el monitoreo mutuo del rendimiento: que el equipo tenga una comprensión compartida de su tarea y de sus responsabilidades, y que su clima sea abierto, de confianza y cohesivo. Es crucial tener un modelo mental compartido, puesto que para practicar el monitoreo del rendimiento cada miembro debe saber cuáles son las responsabilidades de los otros miembros y lo que se supone que deben hacer. El clima de equipo es igualmente importante, porque sin ello sus miembros no podrían comprender los efectos positivos del monitoreo del rendimiento y podrían acaso sentirse sobrecontrolados (McIntyre \& Salas, 1995). Cuando un equipo practica el monitoreo mutuo del rendimiento se recolecta información de cada uno de los miembros a fin de evaluar el rendimiento, identificar errores y detectar incidentes que puedan requerir ayuda. Salas et al. (2005) explican el beneficio de este componente del trabajo en equipo como el proceso de ir de "la suma del rendimiento individual a la sinergia del trabajo en equipo" (p. 576).

- Comportamiento de respaldo 
[Back-up Behavior]. El comportamiento de respaldo ocurre cuando los miembros del equipo proporcionan a los demás recursos y esfuerzo relativo a la tarea, cuando se reconoce que se requiere ayuda porque existe un problema de distribución de la carga de trabajo (Porter, Hollenbeck, Ilgen, Ellis, West \& Moon, 2003). Marks, Mathieu y Zaccaro (2001) identificaron tres formas para proporcionar comportamientos de respaldo: a) vía retroalimentación y coaching; b) vía asistencia física, y c) completando la tarea de un miembro. La retroalimentación y el coaching pueden ser proporcionados para guiar al miembro en el logro de la tarea. Los otros dos medios de respaldo requieren que alguien asuma las actividades del miembro necesitado de ayuda para cumplir la tarea. El comportamiento de respaldo y el monitoreo mutuo de rendimiento están íntimamente relacionados. Un punto importante acerca de los Cinco Grandes es que dichos componentes no son mutuamente excluyentes. Para proporcionar el comportamiento de respaldo, un individuo ha debido monitorear el rendimiento de otro para evaluar si la carga de trabajo se ha incrementado a un nivel mayor que el que este es capaz de realizar.

- Adaptabilidad. Burke, Stagl, Salas,
Pierce y Kendall (2006) consideran la adaptabilidad de equipo como un proceso que le permite ser más eficiente en la búsqueda de sus objetivos. La adaptabilidad ha sido definida como la capacidad de un equipo para reconocer la desviación de los procedimientos de operación normal y hacer las acciones de ajuste necesarias (Priest, Burke, Munim \& Salas, 2002). La adaptabilidad es importante para que los equipos reaccionen ante demandas inesperadas (equipos de atención médica), para tareas que requieren innovación (equipos de investigación y desarrollo), o para equipos que sufren un intento fallido al tratar de cumplir una tarea. Así como el comportamiento de respaldo tiene prerrequisitos, la adaptabilidad también lo requiere. Para mantener una cultura de adaptabilidad se debe tener una perspectiva global de la tarea del equipo, y la habilidad de notar cuando ocurren cambios en el ambiente. De manera similar a los comportamientos de respaldo, la adaptabilidad no es mutuamente excluyente de los otros componentes del trabajo en equipo anteriormente mencionados. Para que un equipo sea adaptable, sus miembros deben estar atentos para identificar rumbos de acción no planeados, cambios en la tarea, y percibir cuándo los procesos actuales son inefi- 
caces y requieren un cambio de acción.

- Orientación de equipo. El último componente del trabajo en equipo contenido en los Cinco Grandes se denomina orientación de equipo. Salas et al. (2005) notaron que los anteriores componentes de los Cinco Grandes eran de naturaleza conductual. Por el contrario, la dimensión de la orientación de equipo es actitudinal. Driskell y Salas (1992) describieron la orientación de equipo no solo como la preferencia para trabajar con otros, sino la tendencia a mejorar el rendimiento individual a través de la coordinación, evaluación y utilización de inputs de los otros miembros mientras se realizan las tareas. Se ha investigado la orientación de equipo y se ha hallado que constituye un importante aspecto del trabajo en equipo. Por ejemplo, la investigación señala que la orientación de equipo mejora el rendimiento individual (Shamir, 1990; Wagner, 1995) así como el rendimiento de equipo (a través del proceso de toma de decisión, Driskell \& Salas, 1992), la satisfacción (Cummings, 1981; Hackman \& Oldham, 1980), y la coordinación y cooperación entre los miembros (Eby \& Dobbins, 1997). La orientación de equipo, aunque no es conductual, es un importante aspecto en la eficacia de equipo y puede disminuir la capacidad de los miembros para exhibir los otros componentes de los Cinco Grandes.

\section{MECANISMOS DE COORDINACIÓN}

Se debe dejar claramente establecido que para que un equipo sea eficaz no es suficiente que se hagan efectivos solo los Cinco Grandes, sino que se tienen que realizar también lo que Salas et al. (2005) llaman mecanismos de coordinación. Estos pueden ser comparables con la noción de transformadores de Shiflett (1979), aunque los mecanismos de coordinación descritos por Salas et al. no determinan cómo los inputs son incorporados, pero aseguran que los Cinco Grandes se realicen y que la información pertinente sea distribuida entre los miembros del equipo. Estos mecanismos de coordinación son los modelos mentales compartidos, la comunicación interconectada y la confianza mutua.

Para que un equipo tenga un modelo mental compartido, sus miembros deben poseer un entendimiento común de las metas del equipo, de sus tareas y sobre la coordinación necesaria para lograr dichas metas (Cannon-Bowers et al., 1995). Existen dos tipos de modelos mentales compartidos que han sido discutidos en la literatura de equipos: los modelos mentales relacionados con el equipo y los vinculados a la tarea (Mathieu, Heffner, Goodwin, Salas \& Cannon-Bowers, 2000). Los modelos mentales relacionados con el 
equipo tienen que ver con el funcionamiento del equipo y las conductas esperadas de los miembros del equipo. Por otro lado, los modelos mentales relacionados con la tarea tienen que ver con la información acerca de los procedimientos y recursos que se requieren para esta. Una forma en que los modelos mentales facilita el trabajo en equipo es promoviendo una comprensión y acción común entre los miembros del equipo (Zaccaro et al., 1995). Si un equipo tiene esta comprensión compartida, se promueven habilidades de trabajo en equipo, como el comportamiento de respaldo y el monitoreo mutuo del rendimiento.

La comunicación interconectada es el intercambio de información entre dos o más individuos (McIntyre \& Salas, 1995; Salas \& Cannon-Bowers, 2000). Debería ser obvio que la comunicación es el medio que mantiene al equipo al día en su progreso hacia el objetivo. La comunicación es también el proceso por el cual los equipos mantienen o actualizan el modelo mental compartido (Salas, Cannon-Bowers \& Johnston, 1996). Es una verdad desafortunada que la comunicación entre los miembros del equipo no siempre ocurre. Por ejemplo, las diferentes razones por las cuales no existe la comunicación se deberían al ruido, el conflicto interpersonal, las barreras lingüísticas o la mala interpretación. La comunicación es un concepto intere- sante para el equipo, y debe realmente alcanzar un equilibrio, entre demasiada comunicación, que podría dificultar el rendimiento del equipo (especialmente en ambientes estresantes, Johnston \& Briggs, 1968), y muy poca comunicación que no proporcione información suficiente para realizar la tarea.

La confianza mutua, el último de los mecanismos de coordinación, es la percepción compartida de que los miembros del equipo realizarán las acciones que son importantes para el equipo, reconocerán los intereses de los miembros y protegerán los intereses del equipo (Webber, 2002). La investigación indica que la confianza tiene un impacto en una variedad de procesos de equipo y los resultados, tales como la participación de grupo y contribución, la calidad del producto y la permanencia de los miembros (Bandow, 2001). También se encuentra que la confianza impacta sobre la comunicación entre los miembros de equipo al promover la disposición para compartir información. Más aún, la confianza mejora la habilidad del equipo para ofrecer comportamientos de respaldo y promueve el monitoreo mutuo del rendimiento, porque con una cultura de confianza los miembros de equipo perciben que dichas acciones son realizadas para el beneficio del equipo. Finalmente, también se necesita confianza para que los miembros acepten al líder y las funciones que este proporciona. Sin la confianza mutua, el 
líder estaría impedido de administrar el equipo eficazmente.

\section{ENTRENAMIENTO DE EQUIPO}

Con el creciente uso de equipos en las organizaciones hay un mayor interés en la mejora de la eficiencia de aquellos. De manera similar a la diversidad de temas encontrados en la literatura sobre equipos, existe también sobre el entrenamiento de estos. La investigación sobre entrenamiento de equipos abarca el desarrollo de técnicas para entrenarlos, revisiones sobre eficacia del entrenamiento de equipos y estudios empíricos con el objetivo de mejorar su efectividad a través del entrenamiento (véase Cannon-Bowers \& Salas, 1998; Dyer, 1984; Salas \& Cannon-Bowers, 2000; Swezey \& Salas, 1992). En esta sección se presenta una breve reseña de las estrategias instruccionales que tiene como objetivo el entrenamiento de equipo. Las estrategias que hemos escogido son aquellas que han sido más usadas e investigadas.

\section{Entrenamiento de coordinación de equipo}

Según el área en que se desarrolle, se le conoce también como entrenamiento de administración de equipo de recursos, entrenamiento de coordinación de tripulación de vuelo, entrenamiento de adaptación y coordinación de equipo. Esta estrategia de entrenamiento es la más común y ha sido utilizada en una diver- sidad de entornos, tales como el de la aviación (Weiner, Kanki \& Helmreich, 1993; Salas, Cannon-Bowers \& Johnston, 1998), el del médico (Gaba, Howard \& Small, 1995; Grogan, Stiles, France, Speroff, Morris, Nixon, Gaffney, Seddon \& Pinson, 2004), militar (Prince \& Salas, 1993; Salas, Bowers \& Cannon-Bowers, 1995) e industrial (Tannenbaum, Beard \& Salas; 1992; Tannenbaum, Salas \& Cannon-Bowers, 1996).

Aun cuando este tipo de entrenamiento tenga varias denominaciones, de acuerdo a las áreas en las que es aplicado, presenta características específicas: 1) se focaliza en la promoción del trabajo en equipo en un entorno particular; 2) se deriva de un marco general del rendimiento de equipo; 3 ) se utilizan diversos métodos, como los basados en la información (conferencias, seminarios), en la demostración (modelaje vía casos sobre comportamientos efectivos e inefectivos), y en la práctica (uso de representación de roles o simulaciones). El propósito fundamental del entrenamiento de coordinación de equipo es enseñar a los miembros de un equipo a usar sus recursos de mejor manera. Los recursos para un mejor uso no se limitan a los materiales o las herramientas, sino principalmente a las personas.

\section{Entrenamiento cruzado}

El entrenamiento cruzado es una estra- 
tegia que proporciona a miembros del equipo experiencias con las tareas, roles y responsabilidades de otros miembros del mismo equipo (Volpe, Cannon-Bowers, Salas \& Spector, 1996). Por ejemplo, puede ser usado en una planta nuclear donde los miembros reciben entrenamiento en diferentes trabajos de la sala de control para comprender todo el proceso del cual es responsable el equipo. Este tipo de entrenamiento se basa en la noción de que los miembros necesitan desarrollar $\mathrm{y}$ mantener estructuras de conocimiento compartido para que funcione el trabajo en equipo (véase Cannon-Bowers, Salas \& Converse, 1993; Stout, Cannon-Bowers \& Salas, 1996). Blickensderfer, Cannon-Bowers \& Salas (1998) sostienen que este tipo de entrenamiento es una estrategia viable para crear las estructuras de conocimiento compartido necesarias para promover y mantener el trabajo de equipo.

\section{Entrenamiento para la autocorrección de equipo}

El entrenamiento para la autocorrección utiliza una variedad de métodos de entrega con el fin de proporcionar a los equipos las habilidades necesarias para reflexionar sobre rendimientos pasados, discutir errores, proporcionar retroalimentación constructiva y planificar una mejora y aprendizaje futuros. La fundamentación teórica de esta estrategia ha sido presentada y puesta a prueba por Blickensderfer, CannonBowers y Salas (1997) y SmithJentsch, Zeisig, Acton y McPherson (1998). En esencia, el entrenamiento para la autocorrección enseña al equipo a utilizar el conocimiento de sus miembros con el propósito de mejorar su rendimiento.

Del mismo modo que todas las estrategias de entrenamiento de equipo, el entrenamiento para la autocorrección puede ser utilizado en diferentes entornos. Por ejemplo, los equipos médicos pueden ser entrenados para analizar sus acciones después de una cirugía o un turno en la sala de emergencias. El uso de la reflexión puede permitir al equipo determinar qué episodios del desempeño ejemplifican conductas eficientes o ineficientes. $\mathrm{Al}$ hacer esto, el equipo adquiere una mejor comprensión de su coordinación y rendimiento, lo que podría asegurar un mejor rendimiento en el futuro.

\section{Entrenamiento basado en la simulación}

Esta estrategia se desarrolló para proporcionar a aquellos equipos que necesitaban practicar sus habilidades (militares, médicos, etcétera), una oportunidad para realizar la tarea durante el entrenamiento. Es importante comprender que practicar una habilidad o realizar una tarea por sí mismas no entrena propiamente a un equipo, sin embargo, la simulación y la práctica debieran estar rodeadas por un conjunto de herra- 
mientas que permita crear un ambiente de aprendizaje. Dicho en forma más simple, el uso de la simulación y práctica debiera estar inmerso en un programa de entrenamiento que permita al equipo practicar en diferentes momentos, lo cual servirá de base para que el equipo pueda evaluar, proporcionar retroalimentación y reflexionar sobre su desempeño. Esta forma de entrenamiento proporciona un enfoque sistemático para entrenar equipos en entornos complejos y dinámicos (Fowlkes, Lane, Salas, Franz \& Oser, 1994).

La reseña de las diferentes estrategias de entrenamiento de equipo proporciona al lector una breve introducción de las estrategias y recursos de entrenamiento de equipo que pueden ser más explorados; invitamos a revisar las numerosas evaluaciones publicadas sobre el tema (Adelman, Christian, Gualtieri \& Bresnick, 1998; Brown, 2003; Cannon-Bowers \& Salas, 1998; Leedom \& Simon, 1995; Pearsall, Ellis $\&$ West, 2004), todas las cuales sugieren que la efectividad mejoró como resultado de la estrategia de entrenamiento de equipo. Más aún, Klein, Salas, DiazGranados, Burke, Stagl, Goodwin, y Halpin (en revisión) realizaron una investigación basada en el meta-análisis acerca de la efectividad del entrena- miento de equipo en la mejora de los procesos de equipo, rendimiento $\mathrm{y}$ resultados afectivos. Concluyeron que el entrenamiento de equipo, en efecto, funciona.

\section{EL FUTURO DE LA INVESTIGACIÓN DE EQUIPOS}

La investigación de equipos ha sido amplia, pero como ocurre en otras ciencias, muchas preguntas quedan sin responder. Los hallazgos disponibles de los estudios pueden dirigir los esfuerzos de los investigadores para encontrar respuestas. Además, existen entornos que recién están aceptando el impacto que tienen los equipos y su entrenamiento sobre los resultados. Por ejemplo, la industria de asistencia médica está empezando a seguir los pasos de la Aviación y el Ejército, al implementar el entrenamiento de equipo (por ejemplo, administración de recursos de equipo, entrenamiento de equipo Stepps) ${ }^{1}$ para convertirse en organizaciones de alta confiabilidad. En los siguientes párrafos discutiremos brevemente algunas de las preguntas sin respuesta que debieran ser el objetivo de la futura investigación.

Las competencias de equipo pueden ser vistas como basadas en conocimiento, habilidad o actitud. Se requiere una 
mayor comprensión de la competencia de equipo basada en el conocimiento. Por ejemplo, sería muy útil una investigación acerca de cómo toman decisiones los equipos. Es importante - para los miembros y los líderes - comprender los procesos cognitivos y colaborativos que experimentan los equipos. Los que actúan en ambientes dinámicos y complejos experimentan una gran cantidad de información durante su tarea; sería beneficioso para ellos conocer mejor cómo procesan información a nivel de equipo y toman decisiones basadas en dicha información.

Se requiere más investigación acerca del papel del líder de equipo. Más específicamente, cómo impacta el líder en el rendimiento y eficacia del equipo. La investigación también podría estar dirigida a comprender mejor de qué manera los comportamientos de liderazgo afectan los procesos de trabajo en equipo, que a su vez impacta en los resultados. Más aún, una investigación más profunda de cómo el líder puede facilitar el proceso de toma de decisión podría permitir una mejor comprensión a los equipos que trabajan en entornos dinámicos y complejos.

Parte importante de las investigaciones son las herramientas utilizadas. Es usual conducir una investigación sobre equipos tomando muestras de estos en un momento determinado, y luego de nuevo en un tiempo posterior. Este tipo de métodos ha sido generalmente la única opción disponible para los investigadores. Es difícil tener acceso y medir las variables relevantes en los equipos de trabajo. Sin embargo, estos obstáculos no debieran impedir la calidad de la investigación ni dificultar la obtención de información disponible sobre equipos. Los investigadores debieran tomar en consideración métodos múltiples para recoger datos de los equipos. Las metodologías cuantitativa y cualitativa debieran ser utilizadas para comprender la complejidad de los equipos en contextos organizacionales. Si bien a la fecha se ha hecho un enorme progreso en la investigación sobre equipos, sin duda esta tendencia continuará. Creemos que estamos en la encrucijada de la investigación sobre equipos, y hacemos eco de la llamada de Mathieu, Maynard, Rapp y Gilson (2008), para un nuevo paradigma de investigación necesario para contestar las preguntas que quedan.

\section{COMENTARIOS FinAles}

En este artículo hemos proporcionado al lector una revisión de los temas más importantes ubicados en la literatura sobre equipos. Se discutió acerca de los tipos de equipos que se encuentran más frecuentemente en las organizaciones y se presentaron los componentes esenciales y los mecanismos de coordinación que debe poseer un equipo para ser eficaz. Los componentes reseñados se conocen generalmente como los Cinco Grandes del trabajo en equipo. 
Además, se revisaron las estrategias para el entrenamiento de equipo para incrementar su eficacia. Se han realizado investigaciones significativas y se espera mucho más en el futuro. No está desapareciendo la tendencia a usar equipos en las organizaciones; en tanto estas los utilicen seguirá la necesidad de más estudios sobre el tema. Es importante para los investigadores ir más allá de lo ya conocido y examinar otras disciplinas y metodologías que puedan ayudarnos a contestar las preguntas que todavía quedan pendientes.

\section{REFERENCIAS}

Adelman, L.; Christian, M.; Gualtieri, J. \& Bresnick, T. A. (1998). Examining the effects of communication training and team composition on the decision making of patriot air defense teams. IEEE Transactions on Systems, Man, and CyberneticsPart A: Systems and Humans, 28(6), 729-741.

Appelbaum, E. \& Batt, R. (1994). The new American workplace: Transforming work systems in the United States. Nueva York: Cornell University.

Bandow, D. (2001). Time to create sound teamwork. Journal for Quality and Participation, 24(2), 41-47.

Blickensderfer, E. L.; Cannon-Bowers, J. A. \& Salas, E. (1997). Training teams to self-correct: An empirical investigation. Trabajo presentado a la 12th Annual Meeting of the Society for Industrial and Organizational Psychology, St. Louis, MO.

Blickensderfer, E.; Cannon-Bowers, J. A. \& Salas, E. (1998). Crosstraining and team performance. En CannonBowers, J. A. \& Salas, E. (Eds.). Making Decisions Under Stress: Implications for Individual and Team Training (pp. 299-311). Washington DC: American Psychological Associates.

Brown, T. C. (2003). The effect of verbal self-guidance training on collective efficacy and team performance. Personnel Psychology, 56, 935-964.

Burke, C. S.; Stagl, K. C.; Salas, E.; Pierce, L. \& Kendall, D. (2006). Understanding team adaptation: A conceptual analysis and model. The Journal of Applied Psychology, 91(6), 1189-1207.

Cannon-Bowers, J. A. \& Salas, E. (1998). Individual and team training under stress: Theoretical underpinnings. En J. A. Cannon-Bowers \& E. Salas (Eds.). Making decisions under stress: Implications for individual and team training (pp. 1738). Washington, DC: American Psychological Association.

Cannon-Bowers, J. A. \& Salas, E. (1998). Making decisions under stress: Implications for individual 
and team training. Washington, DC: American Psychological Association.

Cannon-Bowers, J. A.; Salas, E. \& Converse, S. (1993). Shared mental models in expert team decision making. En Castellan, N. J. (Ed.). Individual and Group Decision Making: Current Issues (pp. 221246). Nueva York: Lawrence Erlbaum.

Cannon-Bowers, J. A.; Tannenbaum, S.; Salas, E. \& Volpe, C. (1995). Defining competencies and establishing team training requirements. En Guzzo, R. R.; Salas, E. \& Asociados (Eds.). Team Effectiveness and Decision Making in Organizations (pp. 333-380). Nueva York: Pfeiffer.

Cohen, S. G. \& Bailey, D. E. (1997). What makes teams work: Group effectiveness research from the shop floor to the executive suite. Journal of Management, 23(3), 239.

Cole, R. E. (1982). Diffusion of participatory work structures in Japan, Sweden, and the United States. En: Goodman, P. S. (Ed.). Change in Organizations: New Perspectives on Theory, Research, and Practice (pp. 230-249). Nueva York: JosseyBass.

Cummings, T. G. (1981). Designing effective work groups. En Nystrom, P. C. \& Starbuck, W. U. Handbook of Organizational Design (Vol. 2, pp. 250-271). Nueva York: Oxford University Press.

Driskell, J. E. \& Salas, E. (1992). Collective behavior and team performance. Human Factors, 34(3), 277-288.

Durkheim, E. (1946). (First published in French, 1893). The Division of Labor in Society. Nueva York: Free Press.

Dyer, J. L. (1984). Team research and team training: A state-of-the-art review. En Muckler, F. A. (Ed.). Human factors review: 1984 (pp. 285-323). Santa Monica, CA: Human Factors Society.

Eby, L. T. \& Dobbins, G. H. (1997). Collectivistic orientation in teams: An individual and group level analysis. Journal of Organizational Behavior, 18(3), 275-295.

Fowlkes, J. E.; Lane, N. E.; Salas, E.; Franz, T. \& Oser, R. (1994). Improving the measurement of team performance: The Targets methodology. Military Psychology, 6(1), 47-61.

Gaba, D. M.; Howard, S. K. \& Small, S. D. (1995). Situation awareness in anesthesiology. Human Factors, 37(1), 20-31.

Goodman, P. S.; Ravlin, E. \& Schminke, M. (1987). Understanding groups in organizations. Research in Organizational Behavior, 9(6), 121-127. 
Grogan, E. L.; Stiles, R. A.; France, D. J.; Speroff, T.; Morris, J. A.; Nixon, B. et al. (2004). The impact of aviation-based teamwork training on the attitudes of health-care professionals. Journal of the American College of Surgeons, 199(6), 843848.

Hackman, J. R. (1986). The psychology of self-management in organizations. En Hackman, J. R. \& Hoffman, L. W. (Eds.). Psychology and Work: Productivity, Change and Employment (pp. 89-136). Washington DC.: American Psychological Association.

Hackman, J. R. \& Oldham, G. R. (1980). Work redesign. Reading, MA: Addison Wesley.

Johnston, W. \& Briggs, G. (1968). Team performance as a function of team arrangement and work load. Journal of Applied Psychology, 52, 89-94.

Katzenbach, J. \& Smith, D. (1993). The wisdom of teams: Creating the high-performance organization. Nueva York: Harper Collins.

Klein, C.; Salas, E.; Diaz Granados, D.; Burke, C. S.; Stagl, K. C.; Goodwin, G. F. \& Halpin, S. M. (under review). Does team training lead to improved team outcomes? A metaanalysis.

Leedom, D. K. \& Simon, R. (1995). Improving team coordination: A case for behavior-based training. Military Psychology, 7(2), 109-122.

Levi, D. (2007). Group dynamics for teams. California: Sage Publications.

Marks, M. A.; Mathieu, J. E. \& Zaccaro, S. J. (2001). A temporally based framework and taxonomy of team processes. Academy of Management Review, 26(3), 356-376.

Mathieu, J.; Maynard, M. T.; Rapp, T. \& Gilson, L. (2008). Team effectiveness 1997-2007: A review of recent advancements and a glimpse into the future. Journal of Management, 34(3), 410.

Mathieu, J. E.; Heffner, T. S.; Goodwin, G. F.; Salas, E. \& Cannon-Bowers, J. A. (2000). The influence of shared mental models on team process and performance. The Journal of Applied Psychology, 85(2), 273-283.

Mayo, E. (1933). The human problems of an industrialized civilization. Nueva York: The MacMillan Company.

McIntyre, R. \& Salas, E. (1995). Measuring and managing for team performance: Emerging principles from complex environments. En Guzzo, R. R.; Salas, E. \& Asociados (Ed.). Team Effectiveness and Decision Making in Organizations (pp. 9-45). Nueva York: Pfeiffer. 
Mohrman, S. A.; Cohen, S. G. \& Morhman, A. M. (1995). Designing team-based organizations: New forms for knowledge work. San Francisco: Jossey-Bass.

Pearce III, J. A. \& Ravlin, E. C. (1987). The design and activation of selfregulating work groups. Human Relations, 40(11), 751.

Pearsall, M. J.; Ellis, A. P. J. \& West, B. J. (August, 2004). Reducing the negative effects of stress in team contexts: The impact of cross-training. Paper presented at the Annual Conference of the Academy of Management, Nueva Orleans, LA.

Porter, C. O.; Hollenbeck, J. R.; Ilgen, D. R.; Ellis, A. P.; West, B. J. \& Moon, H. (2003). Backing up behaviors in teams: The role of personality and legitimacy of need. The Journal of Applied Psychology, 88(3), 391-403.

Priest, H. A.; Burke, C. S.; Munim, D. \& Salas, E. (2002). Understanding team adaptability: Initial theoretical and practical considerations. Proceedings of the Human Factors and Ergonomics Society, 46, 561-565.

Prince, C. \& Salas, E. (1993). Training and research for teamwork in the military aircrew. En Wiener, E. L.; Kanki, B. G. \& Helmreich, R. L. (Eds.). Cockpit resource management (pp. 337-366). San Diego: Academic Press.
Roby, T. B. (1968). Small group performance. Nueva York: Rand McNally.

Roby, T. \& Lanzetta, J. (1957). A replication study of work group structure and task performance. DTIC No. AD, 134, 205. Texas: Air Force Personnel.

Salas, E.; Bowers, C. A. \& CannonBowers, J. A. (1995). Military team research: 10 years of progress. Military Psychology, 7(2), 55-75.

Salas, E.; Burke, C. S. \& CannonBowers, J. A. (2000). Teamwork: Emerging principles. International Journal of Management Reviews, 2(4), 339-356.

Salas, E.; Burke, C. \& Stagl, K. (2004). Developing teams and team leaders: Strategies and principles. En Day, D. V. \& Zaccaro, S. J. (Eds.). Leader Development for Transforming Organizations: Growing Leaders for Tomorrow (pp. 325-355). Mahwah, NJ: Lawrence Erlbaum Associates, Inc.

Salas, E.; Cannon-Bowers, J. A. \& Johnston, J. H. (1998). Lessons lear$\mathrm{n}$ - ed from conducting the TADMUS program: Balancing science, practice and more. En Cannon-Bowers, J. A. \& Salas, E. (Eds.). Making decisions under stress: Implications for individual and team training (pp. 409413). Washington, DC: American Psychological Association. 
Salas, E. \& Cannon-Bowers, J. (2000). The anatomy of team training. En Tobias, S. \& Fletcher, J. D. (Eds.). Training and Retraining: A Handbook for Business, Industry, Government, and the Military (pp. 312335). Nueva York: MacMillan Company.

Salas, E.; Cannon-Bowers, J. \& Johnston, J. (1996). How do you turn a team of experts into an expert team? Emerging training strategies. En Zsambok, C. E. \& Klein, G. (Eds.). Naturalistic Decision Making (pp. 359-370). Illsdale, NJ: Lawrence Earlbaum Associates.

Salas, E.; Dickinson, T. L.; Converse, S. A. \& Tannenbaum, S. I. (1992). Toward an understanding of team performance and training. En Swezey, R. W. \& Salas, R. W. Teams: Their Training and Performance (pp. 3-29). Nueva York: Ablex Publishing.

Salas, E.; Sims, D. E. \& Burke, C. S. (2005). Is there a" big five" in teamwork? Small Group Research, 36(5), 555.

Salas, E. \& Cannon-Bowers, J. A. (2001). The science of training: A decade of progress. Annual Review of Psychology, 52, 471-499.

Shamir, B. (1990). Calculations, values, and identities: The sources of collectivistic work motivation. Human Relations, 43(4), 313.
Shiflett, S. (1979). Toward a general model of small group productivity. Psychological Bulletin, 86(1), 67-79.

Smith-Jentsch, K.; Zeisig, R. L.; Acton, B. \& McPherson, J. A. (1998). Team dimensional training: A strategy for guided team self-correction. En Cannon-Bowers, J. A. \& Salas, E. (Eds.). Making Decisions Under Stress: Implications for Individual and Team Training (pp. 271-297). Washington DC: American Psychological Association.

Stewart, G. L. \& Manz, C. C. (1995). Leadership for self-managing work teams: A typology and integrative model. Human Relations, 48(7), 747.

Stout, R. J.; Cannon-Bowers, J. A. \& Salas, E. (1996). The role of shared mental models in developing team situational awareness: Implications for training. Training Research Journal, 2, 85-116.

Stout, R. J.; Cannon-Bowers, J. A.; Salas, E. \& Milanovich, D. M. (1999). Planning, shared mental models, and coordinated performance: An empirical link is established. Human Factors, 41(1), 61-62.

Sundstrom, E.; DeMeuse, K. \& Futrell, D. (1990). February. American Psychologist, 45(2), 120-133.

Swezey, R. W. \& Salas, E. (1992). Guidelines for use in team-training development. En Swezey, R. W. \& 
Salas, E. (Eds.). Teams: Their Training and Performance (pp. 219-245). Nueva York: Ablex Publishing.

Tannenbaum, S. I.; Salas, E. \& CannonBowers, J. A. (1996). Promoting team effectiveness. En Handbook of Work Group Psychology (pp. 503-529). Nueva York: John Wiley \& Sons.

Taylor, F. W. (1911). The principles of scientific management. Nueva York: Harper and Brothers.

Triplett, N. (1898). The dynamogenic factors in pacemaking and competition. American Journal of Psychology, 9(4), 507-533.

Volpe, C. E.; Cannon-Bowers, J. A.; Salas, E. \& Spector, P. E. (1996). The impact of cross-training on team functioning: An empirical investigation. Human Factors, 38(1), 87-100.
Wagner, J. A. (1995). Studies of individualism-collectivism: Effects on cooperation in groups. Academy of Management Journal, 38(1), 152172.

Webber, S. S. (2002). Leadership and trust facilitating cross-functional team success. The Journal of Management Development, 21(3), 201-214.

Wiener, E. L.; Kanki, B. G. \& Helmreich, R. L. (Eds.). (1993). Cockpit resource management. San Diego: Academic Press.

Wiersema, M. F. \& Bantel, K. A. (1992). Top management team demography and corporate strategic change. Academy of Management Journal, 35(1), 91-121.

Zaccaro, S.; Marks, M.; O'ConnorBoes, J. \& Costanza, D. (1995). The nature and assessment of leader 